\title{
Iterative Joint Channel Estimation and Symbol Detection for Multi-User MIMO OFDM
}

\author{
M. Jiang, J. Akhtman, F. Guo and L. Hanzo ${ }^{1}$ \\ School of ECS, Univ. of Southampton, SO17 1BJ, UK. \\ Tel: +44-703-593 125, Fax: +44-703-593 045 \\ Email: ${ }^{1}$ lh@ecs.soton.ac.uk, http://www-mobile.ecs.soton.ac.uk
}

\begin{abstract}
Multiple-Input-Multiple-Output (MIMO) Orthogonal Frequency Division Multiplexing (OFDM) systems have recently attracted substantial research interest. However, compared to Single-Input-Single-Output (SISO) systems, channel estimation in the MIMO scenario becomes more challenging, owing to the increased number of independent transmitter-receiver links to be estimated. In the context of the Bell LAyered Space-Time architecture (BLAST) or Space Division Multiple Access (SDMA) multi-user MIMO OFDM literature, no channel estimation technique allows the number of users to be higher than the number of receiver antennas, which is often referred to as an "overloaded" scenario. In this contribution we propose a new Genetic Algorithm (GA) assisted iterative joint channel estimation and multiuser detection approach for MIMO SDMA-OFDM systems, which exhibits a robust performance in the above-mentioned overloaded scenario. Furthermore, GA-aided Multi-User Detection (MUD) techniques found in the literature can only provide a hard-decision output, while the proposed GA is capable of providing "soft" outputs, hence achieving an improved performance with the aid of channel decoders. Finally, a range of simulation results are provided to demonstrate the superiority of the proposed scheme.
\end{abstract}

\section{INTRODUCTION}

Multiple-Input-Multiple-Output (MIMO) Orthogonal Frequency Division Multiplexing (OFDM) systems have recently attracted substantial research interest, because the employment of multiple antennas offers an opportunity to exploit both transmitter and receiver diversity, hence significantly increasing the system's transmission integrity. Specifically, intensive research efforts have been invested both in Bell Labs Layered Space-Time architecture (BLAST) and in Space Division Multiple Access (SDMA) based MIMO OFDM [1]. More specifically, in SDMA-OFDM systems the transmitted signals of $L$ simultaneous uplink mobile users - each equipped with a single transmit antenna - are received by the $P$ different receiver antennas of the Base Station (BS). At the BS Multi-User Detection (MUD) techniques are invoked for detecting the different users' transmitted signals with the aid of their unique, user-specific spatial signature constituted by their Frequency-Domain CHannel Transfer Functions (FDCHTFs) or, equivalently, Channel Impulse Responses (CIRs). Since the same time-frequency resource is shared by simultaneous users, a high bandwidth efficiency can be achieved.

However, in these systems accurate channel estimation is required at the receiver for the sake of invoking both coherent demodulation and interference cancellation. Compared to Single-Input-Single-Outpu (SISO) systems, channel estimation in the MIMO scenario becomes more challenging, since a significantly increased number of inde-

Acknowledgements: The financial support of the Mobile VCE is gratefully acknowledged. pendent transmitter-receiver channel links have to be estimated simultaneously for each subcarrier. Moreover, the interfering signals of the other transmit antennas have to be suppressed. In the literature, numerous channel estimation techniques have been proposed for MIMO-OFDM systems, using blind estimation [2], transmitter diversity [3], Constrained Least Squares (CLS) [4], Second-Order Statistics (SOS) based SubSpace (SS) [5] algorithms as well as the QR Decomposition combined with the M-algorithm (QRD-M) [6], etc. However, in the context of the BLAST or SDMA type multi-user MIMO OFDM systems, no channel estimation technique in the literature allows the number of users $L$ to be higher than the number of receiver antennas $P$, which we refer to as an overloaded scenario, where the channel matrix becomes singular and thus rendering the degree of freedom of the detector insufficient.

In the literature, a number of Genetic Algorithm (GA) aided channel estimation schemes have been proposed for Code Division Multiple Access (CDMA) systems [7-9]. However, to our best knowledge, no techniques employing GAs for joint channel and data optimization can be found in the literature in the context of multi-user MIMO OFDM. Furthermore, the GAs found in the literature, for example those of $[7,9,10]$ can only provide a hard-decision output for the Forward Error Correction (FEC) decoder, which inevitably limits the system's achievable performance. Against this background, in this paper we propose a new GA-assisted iterative Joint Channel Estimation and Multi-User Detection (GA-JCEMUD) approach for multi-user MIMO SDMA-OFDM systems, which provides an effective solution to the multi-user MIMO channel estimation problem in the above-mentioned overloaded scenario. Moreover, our proposed GA is capable of providing "soft" outputs and hence achieving an improved performance over conventional GAs.

The structure of this paper is as follows. A detailed analysis of the proposed scheme is given in Section 2, followed by the numerical results of Section 3. Our final conclusions are offered in Section 4.

\section{GA-ASSISTED ITERATIVE JOINT CHANNEL ESTIMATION AND MULTI-USER DETECTION}

The proposed iterative GA-JCEMUD is illustrated in Figure 1. We assume that each OFDM symbol consists of $K$ subcarriers. The first transmitted OFDM symbol of all the $L$ users is completely filled by known pilot Quadrature Amplitude Modulation (QAM) symbols. Within the first OFDM symbol duration $(n=0)$, the BS pilot controller seen in the middle of Figure 1 feeds the pilots to the GAJCEMUD printed in grey, which simultaneously processes the received signals $x_{p}[0, k](p=1, \cdots, P ; k=1, \cdots, K)$ at the $P$ receiver antenna elements. In order to simplify the analysis, we now focus our attention on the $p^{t h}$ receiver. Based on the pilots and the corresponding received signals, the initial estimates of the FD-CHTFs $\tilde{H}_{p}^{(l)}[0, k](l=1, \cdots, L ; k=1, \cdots, K)$ can be generated, followed by the time-domain filtering invoking at the $p^{\text {th }}$ receiver, as plotted on 


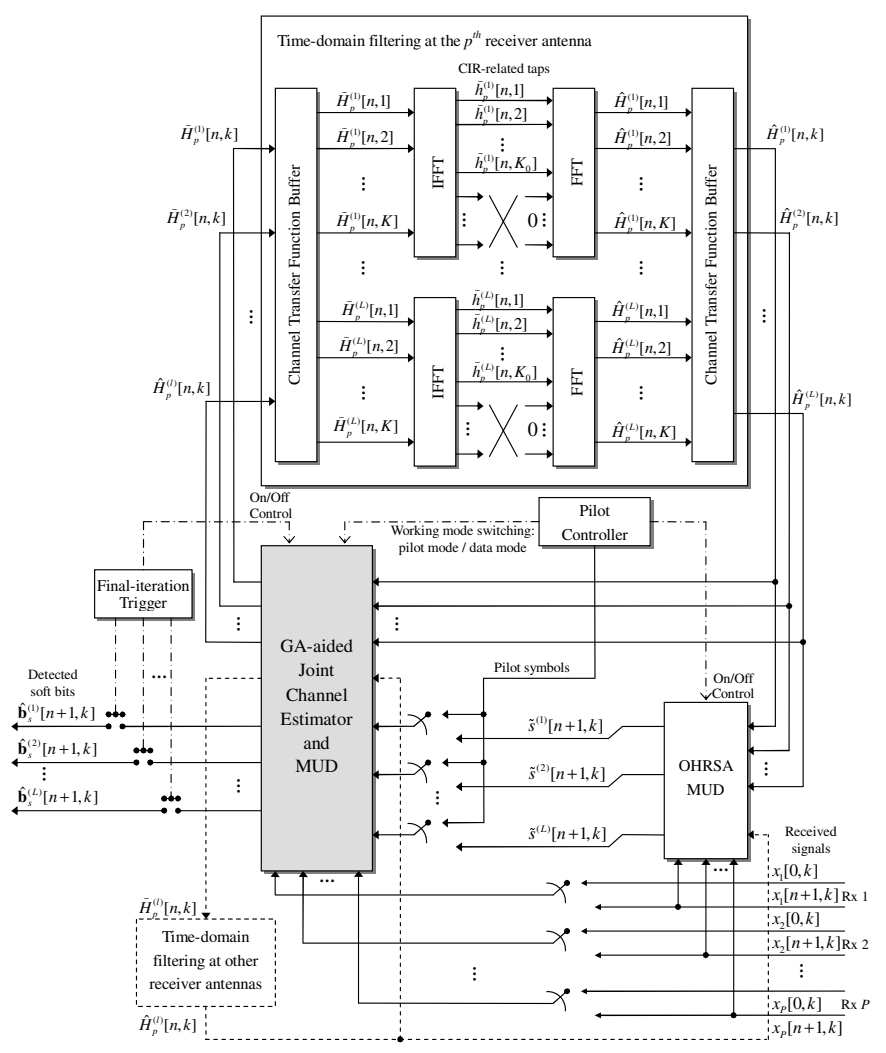

Figure 1: The structure of the proposed iterative GA-JCEMUD.

the top of Figure 1.

The time-domain filtering is conducted for each of the $L$ users on an OFDM symbol basis. More generally, for the $l^{t h}$ user, the $K$ initial FD-CHTF estimates $\tilde{H}_{p}^{(l)}[n, k](k=1, \cdots, K)$ associated with the current, i.e. the $n^{\text {th }}$ OFDM symbol are processed by a $K$-length Inverse Fast Fourier Transform (IFFT), resulting in the set of $K$ uncorrelated CIR-related taps $\tilde{h}_{p}^{(l)}[n, k]$. Then, only the first $K_{0} \mathrm{CIR}$ tap coefficients are retained with the rest set to zero. The value of $K_{0}$ depends on the delay profile of the channel, which is not known a priori at the receiver. However, in many application scenarios it is possible to appropriately over-estimate $K_{0}$ on the basis of previous field experiments. Hence, in our system the value of $K_{0}$ is set to a sufficiently large number so that it significantly exceeds the actual maximum delay spread of the channel. Nonetheless, we point out that the 'significant-tap catching' approaches of [3] can be employed for improving the channel estimator's performance by further removing the low-power taps within the range of $\left[1, \cdots, K_{0}\right]$ according to a pre-defined threshold. The retained CIR-related coefficients $\tilde{h}_{p}^{(l)}[n, k]\left(k=1, \cdots, K_{0}\right)$ are then converted to the improved $a$ postepriori FD-CHTF estimates $\hat{H}_{p}^{(l)}[n, k](k=1, \cdots, K)$ by the Fast Fourier Transform (FFT).

If in the $(n+1)^{t h}$ OFDM symbol duration a data symbol rather than a pilot symbol was transmitted, the pilot controller will enable a first-stage MUD for generating reference symbol estimates. Specifically, the Optimized Hierarchy Reduced Search Algorithm (OHRSA) aided MUD of [11] is employed. With the aid of the a postepriori FD-CHTF estimates $\hat{H}_{p}^{(l)}[n, k]$ associated with the previous OFDM symbol, a subcarrier-by-subcarrier based detection is invoked by the
OHRSA MUD for generating an initial guess of the $L$ users' transmitted symbols $s^{(l)}[n+1, k](l=1, \cdots, L ; k=1, \cdots, K)$. More details of the OHRSA MUD are provided in Section 2.2. The resultant symbol estimates $\tilde{s}^{(l)}[n+1, k]$, the FD-CHTF estimates $\hat{H}_{p}^{(l)}[n, k]$ as well as the corresponding received signals $x_{p}[n+1, k](p=$ $1, \cdots, P ; k=1, \cdots, K)$ are then forwarded to the GA-JCEMUD, where the FD-CHTFs and data symbols associated with the $(n+1)^{t h}$ OFDM symbol are jointly optimized on a subcarrier-by-subcarrier basis, as to be discussed in Section 2.3. The GA-optimized FD-CHTF estimates $\tilde{H}_{p}^{(l)}[n+1, k]$ are then forwarded to the time-domain filters for further enhancement. Based on the improved channel estimates, the OHRSA MUD is now capable of providing a better initial guess of the transmitted symbols for the GA-JCEMUD. This decision-directed process can be invoked for a number of iterations for further performance enhancement. After the final iteration, the $L$ users' detected soft bits $\hat{b}_{s}^{(l)}[n+1, k](l=1, \cdots, L ; k=1, \cdots, K)$ corresponding to the $(n+1)^{\text {th }}$ OFDM symbol are generated.

\subsection{Pilot-aided Initial Channel Estimation}

In order to obtain an initial estimate of the FD-CHTFs, each user's pilot OFDM symbol is multiplied by a user-specific spreading code before it is transmitted. Note that a same unspread pilot QAM symbol can be used by all users, which is known at the receivers. With the aid of the spread pilot OFDM symbols, an initial FD-CHTF estimate is attainable at the receivers, where the Multi-User Interference (MUI) is effectively reduced proportionately to the spreading factor. Note that no spreading is applied to the data OFDM symbols.

Depending on the specific performance-versus-throughput design tradeoff targeted, this process of generating initial channel estimates can be invoked at pre-defined time intervals. Here we denote the pilot overhead as $\epsilon$, which is defined by the ratio of the number of pilot OFDM symbols to the total number of transmitted OFDM symbols. We will show in Section 3 that a good performance is achievable by the proposed scheme with a small value of $\epsilon$.

\subsection{Generating Initial Symbol Estimates}

In the proposed scheme the OHRSA MUD [11] shown in Figure 1 is employed as the first-stage MUD for obtaining initial symbol estimates, with the aid of the a postepriori FD-CHTF estimates generated within the previous OFDM symbol duration. Due to the space limitation, here only a very succinct introduction on the OHRSA MUD is provided.

Briefly speaking, the OHRSA MUD employs a bit-based dendritic recursive search algorithm to find the $L$ users' optimum Maximum Likelihood (ML) estimates. More specifically, two legitimate hypotheses of -1 and 1 are stipulated at each recursive step $i$ of the search algorithm, concerning one of the bits of the trial bitwise vector $\check{\mathbf{b}}_{i}$. The recursive search algorithm is carried on by evaluating a set of inversely ordered Cumulative Sub-Cost (CSC) functions which are derived from the ML metric. For each tentatively assumed value of $\check{\mathbf{b}}_{i}$ a successive recursive search step $(i-1)$ is invoked, which is conditioned on the hypotheses made in all preceding recursive steps $j=i, \cdots, L_{b}=m L$, where $m$ denotes bits per symbol. Upon each arrival at the index $i=1$ of the recursive process, a complete bit-based candidate vector $\check{b}$ associated with a certain symbol vector $\check{\mathrm{s}}$ is hypothesized and the corresponding value of the cost function is evaluated. With the aid of some carefully-designed search rules, the OHRSA MUD is capable of achieving at a near-optimum performance at a significantly-reduced computational complexity. For more details on the OHRSA MUD, the interested reader is referred to [11]. 


\subsection{GA-aided Joint FD-CHTF and Data Optimization Providing Soft Outputs}

\subsubsection{Genetic Evolution}

The basic idea of the GA-based optimization is to find the optimum or a near-optimum solution according to a pre-defined objective function $(O F)$. In the context of joint detection aided SDMA-OFDM systems, the GA's OF associated with the $k^{t h}$ subcarrier in the $n^{t h}$ OFDM symbol can be formulated as:

$$
\Omega(\tilde{\mathbf{H}}[n, k], \tilde{\mathbf{s}}[n, k])=\|\mathbf{x}[n, k]-\tilde{\mathbf{H}}[n, k] \tilde{\mathbf{s}}[n, k]\|^{2},
$$

where $\mathbf{x}[n, k]$ is the received signal vector, while $\tilde{\mathbf{H}}[n, k]$ and $\tilde{\mathbf{s}}[n, k]$ are the trial FD-CHTF matrix and the trial multi-user symbol vector, respectively. Each combination of $\tilde{\mathbf{H}}[n, k]$ and $\tilde{\mathbf{s}}[n, k]$ constitutes a GA individual, which is initialized by the tentative FD-CHTF estimates of Section 2.1 and the initial symbol estimates of Section 2.2, respectively. The GA's ultimate aim is then to find an individual that minimizes the $\mathrm{OF}$ of Equation 1. In comparison to the pure GAbased MUDs [9, 10, 12], which optimize the multi-user data symbols only, the joint optimization work requires the FD-CHTFs to be simultaneously optimized along with the data symbols. Since the FDCHTFs and data symbols are jointly optimized within the same genetic process, the individuals having better FD-CHTF estimates will have a higher probability of producing better symbol estimates and vice versa. Thus, this joint optimization is a "self-adaptive" process with its native intuition leading towards the optimum solution. Furthermore, comparing to other techniques, for example that of [8], where the channel estimation and symbol detection are completed by the GA and the Viterbi Algorithm (VA) separately, the proposed joint scheme is capable of reducing the associated complexity, since the channel estimation is simultaneously achieved with the aid of the same GA process, thus incurring no additional complexity. For more details on the principles of genetic optimization process, we refer to $[7,9,12]$.

However, by the time of writing the GA-aided detection schemes found in the literature, for example those of $[7,9,10,12]$ can only provide single-individual based hard-decoded symbol estimates, which inevitably limits the GA-aided system's attainable performance. In Section 2.3.2, we will introduce a method, which enables the GA to provide soft outputs based on the entire population.

\subsubsection{Generating the GA's Soft Outputs}

At the $k^{t h}$ subcarrier of the $n^{t h}$ OFDM symbol received by the $P$ element receiver antenna array we have the received complex signal vector $\mathbf{x}[n, k]$, which is constituted by the superposition of the independently faded signals associated with the $L$ mobile users and contaminated by the Additive White Gaussian Noise (AWGN), expressed as:

$$
\mathbf{x}[n, k]=\mathbf{H}[n, k] \mathbf{s}[n, k]+\mathbf{n}[n, k],
$$

where $\mathbf{s}[n, k]$ and $\mathbf{n}[n, k]$ are the transmitted and noise signal vectors, respectively, while $\mathbf{H}[n, k]$ is the FD-CHTF matrix. For the sake of notational convenience, the index of $[n, k]$ is omitted in the rest of this section. The soft-bit value or Log-Likelihood Ratio (LLR) associated with the $\left(m_{B}\right)^{t h}$ bit position of the $l^{t h}(l=1, \cdots, L)$ user's transmitted symbol $s^{(l)}$ can be formulated as:

$$
\mathcal{L}_{l, m_{B}}=\ln \frac{P\left(b_{l, m_{B}}=1 \mid \mathbf{x}, \mathbf{H}\right)}{P\left(b_{l, m_{B}}=0 \mid \mathbf{x}, \mathbf{H}\right)},
$$

which is the natural logarithm of the quotient of probabilities that the bit considered has a value of $b_{l, m_{B}}=1$ or $b_{l, m_{B}}=0$. Note that the probability $P\left(b_{l, m_{B}}=b \mid \mathbf{x}, \mathbf{H}\right)$ that the symbol transmitted by the $l^{\text {th }}$ user has the $\left(m_{B}\right)^{t h}$ bit value of $b_{l, m_{B}}=b \in\{0,1\}$, is given by the sum of all the probabilities of the symbol combinations which assume that $b_{l, m_{B}}=b$. Hence, Equation 3 can be equivalently rewritten as:

$$
\mathcal{L}_{l, m_{B}}=\ln \frac{\sum_{\check{\mathbf{s}} \in \mathcal{M}_{l, m_{B}, 1}^{L}} P(\check{\mathbf{s}} \mid \mathbf{x}, \mathbf{H})}{\sum_{\check{\mathbf{s}} \in \mathcal{M}_{l, m_{B}, 0}^{L}} P(\check{\mathbf{s}} \mid \mathbf{x}, \mathbf{H})},
$$

where $\mathcal{M}_{l, m_{B}, b}^{L}$ denotes the specific subset associated with the $l^{\text {th }}$ user, which is constituted by those specific trial vectors, whose $l^{\text {th }}$ element's $\left(m_{B}\right)^{t h}$ bit has a value of $b$, which is expressed as:

$$
\begin{aligned}
\mathcal{M}_{l, m_{B}, b}^{L}= & \left\{\check{\mathrm{s}}=\left[\check{s}^{(1)}, \check{s}^{(2)}, \cdots, \check{s}^{(L)}\right]^{T} \mid\right. \\
& \left.\left\{\check{s}^{(1)}, \check{s}^{(2)}, \cdots, \check{s}^{(L)} \in \mathcal{M}_{c}\right\} \wedge\left\{b_{l, m_{B}}=b\right\}\right\} .
\end{aligned}
$$

With the aid of Bayes' theorem, we have:

$$
P(\check{\mathbf{s}} \mid \mathbf{x}, \mathbf{H})=P(\mathbf{x} \mid \check{\mathbf{s}}, \mathbf{H}) \frac{P(\check{\mathbf{s}})}{P(\mathbf{x})} .
$$

Upon substituting Equation 6 into Equation 4, we arrive at:

$$
\mathcal{L}_{l, m_{B}}=\ln \frac{\sum_{\check{\mathbf{s}} \in \mathcal{M}_{l, m_{B}, 1}^{L}} P(\mathbf{x} \mid \check{\mathbf{s}}, \mathbf{H})}{\sum_{\check{\mathbf{s}} \in \mathcal{M}_{l, m_{B}, 0}^{L}} P(\mathbf{x} \mid \check{\mathbf{s}}, \mathbf{H})} .
$$

Note that here we have assumed that the different $\left(2^{m}\right)$-ary symbol combination vectors $\check{\mathrm{s}}$ have the same probability, namely that $P(\check{\mathbf{s}}), \check{\mathbf{s}} \in \mathcal{M}_{c}$ is a constant. On the other hand, it can be observed from Equation 2 that $\mathrm{x}$ is a random sample of the $L$-dimensional multi-variate complex Gaussian distribution, where the mean vector is $\mathbf{H s}$, while the $(P \times P)$-dimensional covariance matrix $\mathbf{R}_{\mathbf{n}}$ is given by:

$$
\mathbf{R}_{\mathbf{n}}=E\left\{\mathbf{n} \mathbf{n}^{H}\right\}=\sigma_{n}^{2} \mathbf{I},
$$

and the noise encountered at the $P$ number of receiver antennas is assumed to be uncorrelated. Hence, the above-mentioned multi-variate complex Gaussian distribution can be described by:

$$
f(\mathbf{x} \mid \mathbf{s}, \mathbf{H})=\frac{1}{\pi^{P}\left|\mathbf{R}_{\mathbf{n}}\right|} \exp \left\{-(\mathbf{x}-\mathbf{H} \mathbf{s})^{H} \mathbf{R}_{\mathbf{n}}^{-\mathbf{1}}(\mathbf{x}-\mathbf{H} \mathbf{s})\right\} .
$$

When substituting Equation 8 into Equation 9, we have:

$$
f(\mathbf{x} \mid \mathbf{s}, \mathbf{H})=\frac{1}{\pi^{P} \sigma_{n}^{2}} \exp \left\{-\frac{1}{\sigma_{n}^{2}}\|\mathbf{x}-\mathbf{H} \mathbf{s}\|^{2}\right\} .
$$

Note that $f(\mathbf{x} \mid \mathbf{s}, \mathbf{H})=P(\mathbf{x} \mid \mathbf{s}, \mathbf{H})$ is the a priori probability that the vector $\mathbf{x}$ has been received under the condition that the vector $\mathbf{s}$ was transmitted over the MIMO channel characterized by the FD-CHTF matrix $\mathbf{H}$. Thus, Equation 7 can be further developed with the aid of Equation 10, yielding:

$$
\mathcal{L}_{l, m_{B}}=\ln \frac{\sum_{\check{\mathbf{s}} \in \mathcal{M}_{l, m_{B}, 1}^{L}} \frac{1}{\pi^{P} \sigma_{n}^{2}} \exp \left\{-\frac{1}{\sigma_{n}^{2}}\|\mathbf{x}-\mathbf{H} \check{\mathbf{s}}\|^{2}\right\}}{\sum_{\check{\mathbf{s}} \in \mathcal{M}_{l, m_{B}, 0}^{L},} \frac{1}{\pi^{P} \sigma_{n}^{2}} \exp \left\{-\frac{1}{\sigma_{n}^{2}}\|\mathbf{x}-\mathbf{H} \check{\mathbf{s}}\|^{2}\right\}} .
$$

In order to avoid the exponential computation imposed by Equation 11, the maximum-approximation [1] can be applied, yielding:

$$
\mathcal{L}_{l, m_{B}} \approx-\frac{1}{\sigma_{n}^{2}}\left[\left\|\mathbf{x}-\mathbf{H}_{l, m_{B}, 1}\right\|^{2}-\left\|\mathbf{x}-\mathbf{H} \check{\mathbf{s}}_{l, m_{B}, 0}\right\|^{2}\right],
$$


where

$$
\check{\mathbf{s}}_{l, m_{B}, b}=\arg \left\{\min _{\check{\mathbf{s}} \in \mathcal{M}_{l, m_{B}, b}^{L}}\left[\|\mathbf{x}-\mathbf{H} \check{\mathbf{s}}\|^{2}\right]\right\}, \quad b=0,1 .
$$

Furthermore, concerning the fact that the true FD-CHTF matrix $\mathbf{H}$ is unknown and using Equation 1, Equation 12 can be represented as:

$$
\mathcal{L}_{l, m_{B}} \approx-\frac{1}{\sigma_{n}^{2}}\left[\bar{\Omega}_{l, m_{B}, 1}-\bar{\Omega}_{l, m_{B}, 0}\right],
$$

where

$$
\bar{\Omega}_{l, m_{B}, b}=\min \left[\Omega\left(\tilde{\mathbf{H}}, \check{\mathbf{s}}_{l, m_{B}, b}\right), \omega\right], \quad b=0,1,
$$

and $\omega=P \cdot L$ is a normalization factor. Equation 14 suggests that the LLRs can be obtained by evaluating the GA's OF. More explicitly, in order to calculate the LLR of the $\left(m_{B}\right)^{t h}$ bit of the $l^{t h}(l=1, \cdots, L)$ user at the specific subcarrier considered, the $X$ number of individuals in the GA's final generation are divided into two groups, where the first (or second) group is constituted by those individuals that have a value of one (or zero) at the $\left(m_{B}\right)^{t h}$ bit of the $l^{\text {th }}$ user's estimated transmitted symbol. The resultant lowest OS calculated in each of the two groups is then compared to $\omega$, and the smaller of the two will be used in Equation 14 for calculating the corresponding LLR, which can therefore assist the channel decoder in improving the SDMA-OFDM system's performance.

It is worth pointing out that the proposed GA generating the abovementioned population-based soft outputs only imposes a modest complexity increase in comparison to the conventional hard-decision aided individual-based GAs $[7,9,10,12]$. This is because the only additional operation required by the proposed scheme is to compare $\omega$ to the results of the OF evaluation carried out by the conventional GAs.

\section{SIMULATION RESULTS}

In this section, we will quantify the performance of the MIMO SDMAOFDM system using the proposed iterative GA-JCEMUD technique. Due to space limitations, here we only provide the results achieved in an overloaded scenario associated with $L=4$ users and $P=2$ receiver antenna elements. However, we point out that the proposed scheme also performs well in the scenarios associated with $L \leqslant P$.

An OFDM modem employing $K=64$ subcarriers and a cyclic prefix of eight samples was used. A two-path Rayleigh fading channel model was employed, where each path experienced independent fading having the same Doppler frequency of $F_{D}$ normalized to the OFDM symbol rate. The delay profile of the channel was $(0.7740$. $\left.z^{0}+0.6332 \cdot z^{-1}\right)$. The value of the parameter $K_{0}$ was set to $8 \gg 2$, which is potentially capable of tolerating an increase of the actual dispersion up to eight CIR taps. The complex-valued channel envelope was assumed to remain unchanged within an OFDM symbol duration. The fading envelope of the $P \times L$ number of user-receiver channel links were assumed to be uncorrelated. Each user's associated transmit power or signal variance was assumed to be unity.

Both scenarios with and without FEC coding were investigated. In the FEC-coded scenario, as an example, a half-rate binary Low Density Parity Check (LDPC) [13] code was employed, which assumed a block length of 640 input bits, a column weight of 2.5 and a maximum of 10 decoding iterations. It is worth pointing out that other FEC codes, for example turbo codes are also applicable to the proposed system. Furthermore, the GA's configuration was similar to that of [12], except that the method of [7] was used for FD-CHTF mutation.

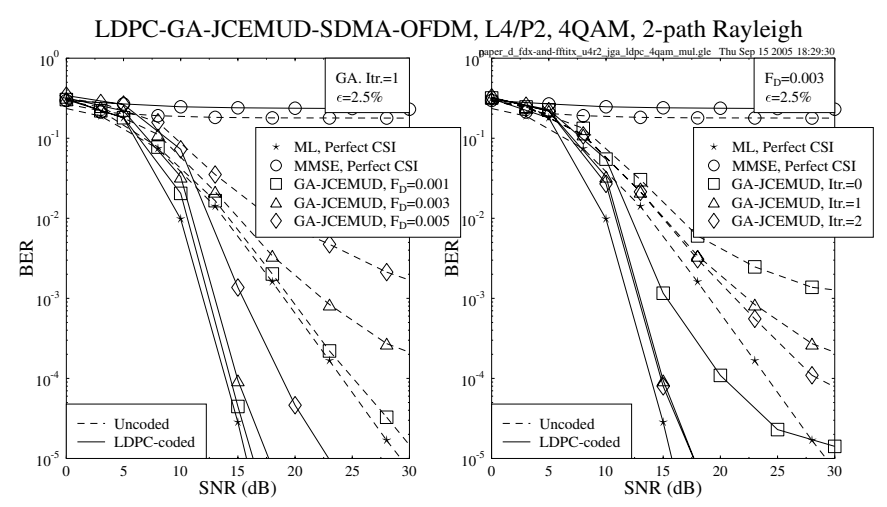

Figure 2: BER versus SNR performances of the uncoded and LDPCcoded iterative GA-JCEMUD/SDMA-OFDM systems at different $F_{D}$ (left) and different GA-JCEMUD iterations (right).

The Bit Error Ratio (BER) versus Signal-to-Noise Ratio (SNR) performances of both the uncoded and LDPC-coded GA-JCEMUD assisted SDMA-OFDM systems recorded at different values of $F_{D}$ and different numbers of GA-JCEMUD iterations are provided at the left-hand and right-hand sides of Figure 2, respectively. The performances of the systems employing the linear MMSE MUD or the optimum ML MUD are also provided as references, both assuming perfect Channel State Information (CSI). A pilot overhead of $\epsilon=2.5 \%$ was assumed and the GA-JCEMUD used a single iteration. As seen at the left-hand side of Figure 2, the performances of the GA-JCEMUD aided systems degraded, when $F_{D}$ was increased, since channel estimation becomes more challenging when the channel fades more rapidly. This is especially true for MIMO systems, even more so for overloaded MIMO systems, as discussed in Section 1. Nonetheless, with only a $2.5 \%$ pilot overhead, the proposed GA-JCEMUD/SDMAOFDM system was capable of achieving a performance close to the perfect-CSI aided optimum ML MUD at $F_{D}=0.001$. By contrast, the system employing the MMSE MUD completely failed even with the aid of perfect CSI, owing to the insufficient degree of detection freedom experienced in overloaded scenarios. On the other hand, a significant iteration gain was achievable, when the GA-JCEMUD invoked more iterations, as revealed by the right-hand side of Figure 2, where we had $\epsilon=2.5 \%$ and $F_{D}=0.003$.

In Figure 3 the BER performance of the proposed system using different pilot overheads is investigated. In most cases, the GAJCEMUD was capable of achieving a good performance using a pilot overhead as low as $\epsilon=1.5 \sim 2.5 \%$. Furthermore, the increase of pilot OFDM symbol overhead brings about more substantial benefits at the higher Doppler frequencies than at the lower ones, especially in the scenarios associated with higher SNRs, where an increasing fraction of the residual detection errors was inflicted by inaccurate channel estimation.

In order to further characterize the advantages of the proposed joint optimization scheme, on the left-hand side of Figure 4 we compare the performances of the GA-JCEMUD and its counterpart, referred to as the GA-based channel estimator assisted OHRSA MUD (GACE-OHRSA-MUD), where the OHRSA MUD is serially concatenated with the stand-alone GA-aided channel estimator. More specifically, in the GACE-OHRSA-MUD arrangement the symbol estimates offered by the OHRSA MUD are fixed during the GA-aided optimization process of the FD-CHTF estimates. Explicitly, in this case the effect of error propagation due to inaccurate symbol and/or 
LDPC-GA-JCEMUD-SDMA-OFDM, L4/P2, 4QAM, 2-path Rayleigh

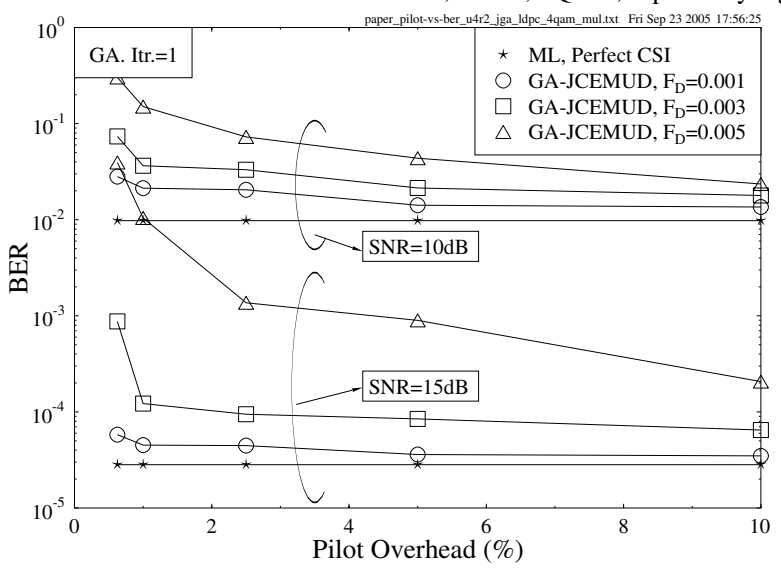

Figure 3: BER versus pilot overhead performance of the LDPC-coded iterative GA-JCEMUD/SDMA-OFDM system at different $F_{D}$.
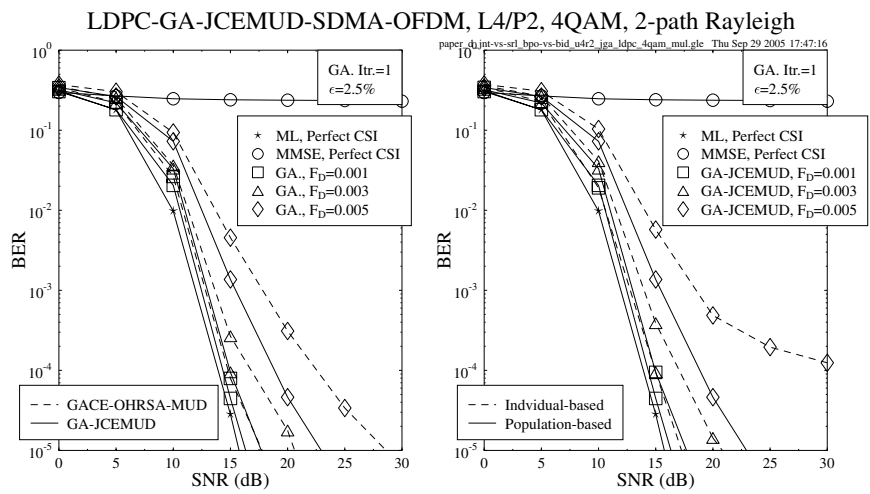

Figure 4: Performance comparison of the LDPC-coded SDMAOFDM system using either the iterative GA-JCEMUD or the GACEOHRSA-MUD (left) and using either the conventional individualbased or the proposed population-based GA-JCEMUD (right) at different $F_{D}$.

channel estimates will become more severe, thus resulting in a dramatic BER performance degradation in comparison to the proposed joint optimization scheme.

Last but not least, we present the performance comparison of the GA-JCEMUD providing either the conventional individual-based hard outputs $[7,9,10,12]$ or the proposed population-based soft outputs, as shown on the right-hand side of Figure 4. As expected, with the advent of FEC codes, the proposed soft GA is capable of significantly outperforming the conventional arrangement, especially when the channel fades more rapidly. This result implies the proposed GA is more robust against fast fading channels than the conventional GAs.

\section{CONCLUSIONS}

From the investigations and discussions conducted, we conclude that the proposed iterative GA-JCEMUD technique generating soft outputs constitutes an effective solution to the channel estimation problem in multi-user MIMO SDMA-OFDM systems. Furthermore, it is capable of exhibiting a robust performance in overloaded scenarios, where the number of users is higher than the number of receiver antenna elements, either with or without FEC coding. This attractive property enables the SDMA-OFDM system to potentially support an increased number of users.

\section{REFERENCES}

[1] L. Hanzo, M. Münster, B. J. Choi, and T. Keller, OFDM and MC-CDMA for Broadband Multi-user Communications, WLANs and Broadcasting. IEEE Press - John Wiley \& Sons Ltd., 2003.

[2] M. C. Necker and G. L. Stüber, "Totally blind channel estimation for OFDM on fast varying mobile radio channels," IEEE Transactions on Wireless Communications, vol. 3, pp. 15141525, September 2004.

[3] Y. Li, N. Seshadri, and S. Ariyavisitakul, "Channel Estimation for OFDM Systems With Transmitter Diversity in Mobile Wireless Channels," IEEE Journal on Selected Areas in Communications, vol. 17, pp. 461-471, March 1999.

[4] S. Thoen, L. Deneire, L. V. der Perre, M. Engels, and H. D. Man, "Constrained Least Squares Detector for OFDM/SDMA-based Wireless Networks," IEEE Transactions on Wireless Communications, vol. 2, pp. 129-140, January 2003.

[5] Y. Zeng and T. S. Ng, "A Semi-blind Channel Estimation Method for Multiuser Multiantenna OFDM Systems," IEEE Transactions on Signal Processing, vol. 52, pp. 1419-1429, May 2004.

[6] K. J. Kim, J. Yue, R. A. Iltis, and J. D. Gibson, “A QRDM/Kalman Filter-based Detection and Channel Estimation Algorithm for MIMO-OFDM Systems," IEEE Transactions on Wireless Communications, vol. 4, pp. 710-721, March 2005.

[7] K. Yen and L. Hanzo, "Genetic algorithm assisted joint multiuser symbol detection and fading channel estimation for synchronous CDMA Systems," IEEE Journal on Selected Areas in Communications, vol. 19, pp. 985-998, June 2001.

[8] S. Chen and Y. Wu, "Maximum likelihood joint channel and data estimation using genetic algorithms," IEEE Transactions on Signal Processing, vol. 46, pp. 1469-1473, May 1998.

[9] L. Hanzo, L.-L. Yang, E.-L. Kuan, and K. Yen, Single- and Multi-Carrier DS-CDMA: Multi-User Detection, Space-Time Spreading, Synchronisation and Standards. IEEE Press - John Wiley \& Sons Ltd., 2003.

[10] C. Ergün and K. Hacioglu, "Multiuser Detection Using a Genetic Algorithm in CDMA Communications Systems," IEEE Transactions on Communications, vol. 48, pp. 1374-1383, August 2000.

[11] J. Akhtman and L. Hanzo, "Reduced-Complexity MaximumLikelihood Detection in Multiple-Antenna-Aided Multicarrier Systems," in Proceedings of the 5th International Workshop on Multi-Carrier Spread Spectrum Communications, (Oberpfaffenhofen, Germany), 14-16 September 2005. Available at http://www.ecs.soton.ac.uk/ mj02r/t/mcss-05.pdf.

[12] M. Jiang and L. Hanzo, "Improved Hybrid MMSE Detection for Turbo Trellis Coded Modulation Assisted Multi-User OFDM Systems," IEE Electronics Letters, vol. 40, pp. 1002-1003, August 2004.

[13] R. Gallager, "Low Density Parity Check Codes," IEEE Transactions on Information Theory, vol. 8, pp. 21-28, January 1962. 